\title{
Forward and backward recall in the suffix paradigm
}

\author{
SUSAN KARP MANNING \\ Hunter College, City University of New York, New York, New York 10021
}

\begin{abstract}
If stimuli are presented auditorily and an auditory suffix is appended to a sequence, the suffix produces a decrement in forward serial recall that is specific to the end of the sequence, that is, to the items nearest the suffix. Sensory trace theory explains this decrement as being a result of lost access to sensory traces remaining after the sequence has ended. In the current experiment, subjects recalled sequences with and without a suffix in backward and forward order. The backward recall results showed that the suffix produced a generalized decrement. The fact that this decrement was not specific to the last presented/first recalled items casts doubt on the sensory trace explanation of the end-of-sequence decrement in auditory forward serial recall.
\end{abstract}

A suffix may be defined as an extra, not-to-be-recalled item appended to a series of items to be recalled. Although the suffix does not need to be either stored or recalled, its presence results in a decrement in the recall of the items in the sequence. Although the suffix is rarely found in everyday recall tasks, understanding its decremental effect is important to our concept of shortterm recall. Why should something that need not be recalled hinder the recall of other items?

Various types of suffix effects have been identified. For example, visual suffixes with visual sequences produce performance decrements of different sorts (see Kahneman, 1973; Manning, 1980). Tactual suffix effects have been reported (e.g., Manning, 1980).

However, one particular type of suffix effect has received a great deal of attention. This is the consistent and strong finding that an auditory suffix leads to a performance decrement that is primarily localized at the end of a recalled sequence, that is, mainly limited to the items nearest the suffix. [It should be noted, however, that smaller decrements may occur with auditory stimuli and suffixes at earlier parts of the sequence (e.g., Penney, 1978).]

The earliest major theory concerned with the end-ofsequence effect was developed by Crowder and Morton (1969) and was recently updated by Crowder (1978). These researchers postulated that sensory traces linger for 1 or $2 \mathrm{sec}$ after the cessation of the stimulus. These sensory traces, by providing additional information, result in extra rehearsal of the more recent items before

This research was supported by Grant BNS-76-18683 from the National Science Foundation to Susan Karp Manning. A version of this paper was presented at the meetings of the American Psychological Association, Los Angeles, California, in August 1981. I wish to thank Martin Chodorow for his help. Please address reprint requests to Susan Karp Manning, Department of Psychology, Hunter College, 695 Park Avenue, New York, New York 10021. the start of recall. This rehearsal is hypothesized to lead to the large recency effect frequently found in the recall of auditorily presented numbers, letters of the alphabet, and syllables. In the presence of an auditory suffix from one of the above sets of stimuli, access to the lingering sensory traces is thought to be reduced, resulting in the large end-of-sequence decrement.

A series of studies has cast serious doubts on whether this end-of-sequence effect is due to sensory traces or to some other mechanism. For example, Spoehr and Corin (1978) have shown that when sequences of digits are to be recalled, a mouthed "zero" leads to a suffix effect that is virtually indistinguishable from a spoken "zero." Visual presentation of "0" or "zero" does not produce such results.

Additionally, Shand and Klima (1981) reported U-shaped serial position curves and end-of-sequence suffix effects for congenitally deaf subjects who were tested using sequences and suffixes composed of signs used in American Sign Language.

Thus, suffix effects that are apparently the same as those caused by auditory suffixes on auditory sequences are caused by other types of stimuli. In the study by Spoehr and Corin (1978), these effects were shown even on auditorily presented digits. The question therefore remains as to whether there is, in fact, any sensory trace component to the special end-of-sequence effect.

In order to answer this question, a new suffix paradigm was developed. Subjects were given a sequence of digits followed by a suffix (e.g., 3997466310$)$ and asked to recall the items in reverse order (e.g., 1364793 ). Recall was compared for a control condition without a suffix and for standard forward recall conditions, with and without a suffix.

If the standard forward recall suffix effect is caused by interference with sensory traces, the decrement in backward as well as in forward recall caused by the suffix should be on those items nearest the suffix. In 
the backward recall paradigm, these are the last presented/ first recalled items.

In addition to testing the prediction of sensory trace theory with respect to the location of the suffix effect, this experiment adds further information relevant to another major interpretation of the effects of a suffix. Kahneman (1973), in his attentional interpretation, has suggested that the suffix functions as an extra item in overloading short-term memory storage. Support for this overload hypothesis is provided by Parkinson and Hubbard (1974), who reported suffix effects for subjects with a shorter digit span but not for those with a longer digit span. However, when subjects with the longer digit span were presented with sequences of increased length, they also showed suffix effects.

Thus, in addition to seven-digit control sequences and seven-digit sequences with a suffix, eight-digit control sequences were included and compared to the suffix condition. In both forward and backward recall conditions, however, because the comparison of different length sequences cannot be made simply (should one compare the first or last presented of eight digits to seven-digit sequences?), analyses using the mean scores per digit were performed.

\section{METHOD}

\section{Subjects}

The subjects were 32 volunteer Hunter College students whose first language was English.

\section{List Construction and Experimental Design}

Two lists, A and B, were constructed. Each consisted of 18 sequences: 6 seven-unit control sequences, 6 seven-unit sequences followed by the suffix " 0 ," and 6 eight-unit control sequences. The three conditions were arranged in a random block design in each list.

Sequences were randomly generated with the restriction that they contain no more than two digits following in succession and that they contain no obvious patterns.

Two additional lists, $A^{\prime}$ and $B^{\prime}$, were formed by reversing all the sequences in Lists $A$ and $B$. Thus, if the sequence 9631 472 was presented in $A$, the sequence 2741369 was presented in $A^{\prime}$.

Each subject received one of eight conditions: $A$ followed by $A^{\prime}$ or $B^{\prime}, A^{\prime}$ followed by A or B, B followed by $A^{\prime}$ or $B^{\prime}$, and $B^{\prime}$ followed by $A$ or $B$. Thus, only half of the 16 possible combinations of two lists were presented, an error due to the incorrect programming of the research assistant. All subjects were asked to recall $A$ or $B$ in forward order and $A^{\prime}$ or $B^{\prime}$ in backward order. Thus, a subject tested on forward recall first was given a backward recall test second and vice versa. The conditions were also incorrectly balanced, so that $\mathrm{N}=6$ for $\mathrm{A}$ first, $\mathrm{N}=10$ for B first, $\mathrm{N}=7$ for $\mathrm{A}^{\prime}$ first, and $\mathrm{N}=9$ for $\mathrm{B}^{\prime}$ first. Analyses for differences due to order showed no significant effects. Thus, errors should not have affected the experimental results.

\section{Apparatus and Procedure}

Four tapes were made, with a female reading the stimuli. These tapes were played at a contant volume on a Sony TC 377 reelto-reel, four-track, two-channel tape deck with a McMartin LX40A preamplifier. The stimuli were presented monaurally to the subject's left ear. Digits were presented at a rate of $1 \mathrm{unit} / \mathrm{sec}$ with a $20 \mathrm{sec}$ interval between sequences for the responses.
Subjects were told that a prerecorded tape would be played. They were to recall aloud in either forward or backward order 18 sequences of the numbers 1-9. They were instructed that when a sequence was followed by a 0 , the 0 was to be omitted from recall. After the subject completed the first condition, a short break was given, followed by appropriate instuctions for the second condition.

\section{RESULTS}

Data were scored in such a way that a response was considered correct if and only if it appeared in recall both in the order and position required. Figure 1 presents the mean number of digits recalled in each position in the seven-unit forward and backward recall conditions, with and without the suffix. Because six sequences of each type were presented, the maximum number of correct responses at each position was six.

Examination of these data shows a typical U-shaped serial position curve for the forward recall control condition. For the analogous backward recall condition, the last presented items show a high degree of recency followed by a fall in performance. The means for these conditions, as well as for the eight-unit control, can be seen in Table 1.

Analysis of the effects of the suffix on errors in the seven-unit conditions was performed using a 2 (recall order) by 2 (suffix vs. control) by 7 (serial positions) analysis of variance. This and all other statistics are reported at the .05 level of significance. The main effects of recall order $[\mathrm{F}(1,31)=61.06, \mathrm{MSe}=4.35]$, suffix $[\mathrm{F}(1,31)=21.66, \quad \mathrm{MSe}=2.19]$, and serial position $[\mathrm{F}(6,186)=44.96, \mathrm{MSe}=2.19]$ were all significant due to, respectively, the inferior performance of backward as

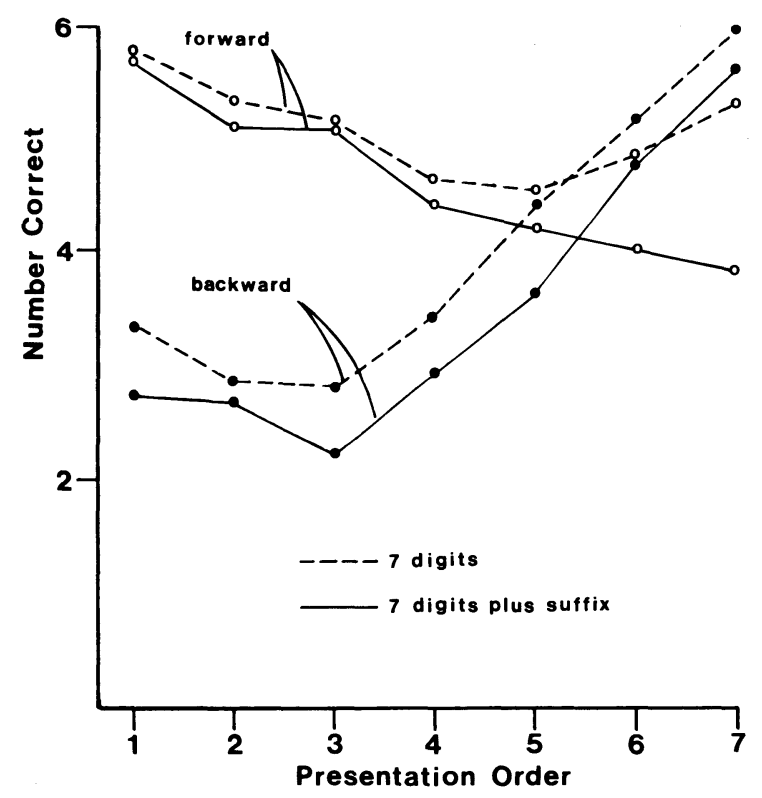

Figure 1. The mean number of items recalled for the sevenunit conditions. 
Table 1

Mean Number of Items Recalled for the Various Conditions

\begin{tabular}{|c|c|c|c|c|c|c|c|c|c|}
\hline & \multicolumn{9}{|c|}{ Presentation Position } \\
\hline & 1 & 2 & 3 & 4 & 5 & 6 & 7 & 8 & Mean \\
\hline & \multicolumn{9}{|c|}{ Backward Recall } \\
\hline Seven-Unit Control & 3.34 & 2.88 & 2.81 & 3.41 & 4.41 & 5.25 & 6.00 & & 4.01 \\
\hline Seven-Unit Suffix & 2.78 & 2.69 & 2.25 & 2.94 & 3.62 & 4.84 & 5.66 & & 3.54 \\
\hline \multirow[t]{2}{*}{ Eight-Unit Control } & 2.25 & 1.72 & 1.72 & 2.44 & 3.47 & 4.44 & 5.44 & 5.94 & 3.43 \\
\hline & \multicolumn{9}{|c|}{ Forward Recall } \\
\hline Seven-Unit Control & 5.79 & 5.34 & 5.16 & 4.62 & 4.53 & 4.88 & 5.31 & & 5.09 \\
\hline Seven-Unit Suffix & 5.72 & 5.12 & 5.12 & 4.44 & 4.22 & 4.00 & 3.88 & & 4.64 \\
\hline Eight-Unit Control & 5.69 & 5.28 & 5.00 & 4.66 & 4.12 & 3.97 & 4.00 & 4.91 & 4.70 \\
\hline
\end{tabular}

Note-The maximum number of correct responses at each position is six.

compared with forward recall, the suffix condition as compared with the control condition, and the various irregularities of serial position.

The interaction of Recall Order by Suffix was not significant $(F<1)$, indicating that the size of the suffix effect was approximately the same for both recall orders. The interactions of Recall Order by Serial Position $[\mathrm{F}(6,186)=16.59, \mathrm{MSe}=1.07]$ and Suffix by Serial Position $[\mathrm{F}(6,186)=3.79, \mathrm{MSe}=.57]$ were significant.

Of more common interest, however, was the significant interaction of Recall Order by Suffix by Serial Position $[\mathrm{F}(6,186)=3.33$, MSe $=.82]$. As can be seen in Figure 1, although some consistent drop throughout the serial position curve may be noted, the effect of the suffix in forward recall is clearly greatly increased at the end as compared with the beginning of the presented list. For the backward condition, however, the serial position curves for the suffix and control conditions are virtually parallel.

Additionally, an effort was made to evaluate whether the suffix functions in a way similar to an extra item, as has been suggested by Kahneman (1973) and others. To do this, the mean score per digit was calculated for the suffix and the seven- and eight-unit control conditions for backward and forward recall. These means are shown in Table 1.

These means are useful in determining the effect of an extra item on performance, because when comparisons are made between sequences of differing lengths, it is not clear which parts of the longer sequence should be compared with the shorter one (i.e., would Positions $1-7$ or 2.8 of an eight-unit sequence provide the appropriate comparison with a seven-unit sequence?).

The comparisons of the means of the seven-unit control to the means of the seven-unit suffix condition were largely redundant with the previous analysis and will not be reported here. A 2 (recall order) by 2 (seven-unit suffix condition vs. eight-unit control) analysis of variance was performed in order to determine whether a significant difference was present in the mean per digit for the eight-unit control as compared with the suffix condition. The results of this analysis showed a significant effect of recall order $[\mathrm{F}(1,31)=58.06, \mathrm{MSe}=.78]$.
Of interest, however, were the findings that neither the the main effect of condition nor the interaction of Recall Order by Condition was reliable (both $F_{s}<1$ ). The demonstration that there was no significant difference between the mean per digit of the suffix and the eight-unit control, in the absence of a significant interaction, clearly suggests that the suffix caused decrements of the same size as an extra item for both recall orders.

\section{DISCUSSION}

This experiment adds some new information to the highly complex literature on suffix effects. The results of this work on the backward recall paradigm can now be added to the increasing literature that casts doubts on the sensory trace view of the auditory suffix effect. The sensory trace view predicts that those stimuli closest to the suffix will be affected by its presence. Although there was an effect on the last presented/first recalled stimuli, it was no greater than that at most other positions.

Thus, it is clear that the expectations of the theory were not borne out. Further, unlike in the experiments of Shand and Klima (1981) and Spoehr and Corin (1978), both stimuli and suffixes were presented auditorily. In those two studies, nonauditory stimuli performed as auditory stimuli. Here auditory stimuli failed to fulfill the predictions of sensory trace theory.

What these results suggest is that the auditory suffix effect in forward recall, although easy to obtain and in some sense unique, is probably not caused by sensory traces. If it were, the backward recall results would be different.

What then might be happening in the backward recall paradigm? Rudel and Denckla (1974) have suggested that subjects may be arraying the stimuli in some sort of spatial pattern in order to facilitate recall. Whether this or some other explanation of how backward recall is accomplished is correct, one very likely possibility is that the mechanisms in backward recall differ from those in forward recall. Thus, in all probability, the source of the end-of-sequence suffix effect in forward recall lies in some strategic aspect of forward recall that is not present in backward recall. ${ }^{1}$

What then is the probable source of the suffix effects we have obtained? While we have no definite answers, at least some aspects of suffix effects are probably caused by the suffix taking up space in a limited-capacity short-term memory store. This view is supported by our analyses of the mean-per-digit scores. In both forward and backward recall, the mean per digit in the suffix condition is virtually the same as in the eight-unit control, and less than in the seven-unit control.

Finally, we did not use a delayed recall control group. As a result, in both forward and backward recall, the suffix conditions were delayed in recall $1 \mathrm{sec}$ more than the seven-unit con- 
trols. We did not use this control because of the difficulty in finding an appropriate signal. While Crowder and many others have used tones, there is evidence in some data presented by Crowder (1978) that at least at the earlier parts of the recall curve, such "signals" may be leading to performance decrements of some size. Thus, we chose to allow the small time differential instead. Because there is no evidence that our forward suffix results are in any sense unusual or that small unfilled delays affect recall (Baddeley \& Hull, 1979), we doubt that this condition had any effect.

\section{REFERENCES}

Baddeley, A., \& Hull, A. Prefix and suffix effects: Do they have a common basis? Journal of Verbal Learning and Verbal Behavior, 1979, 18, 129-140.

Crowder, R. G. Mechanisms of auditory backward masking in the stimulus suffix effect. Psychological Review, 1978, 85, 502524.

Crowder, R. G., \& Morton, J. Precategorical acoustic storage (PAS). Perception \& Psychophysics, 1969, 6, 365-373.

Kahneman, D. Attention and effort. Englewood Cliffs, N.J: Prentice-Hall, 1973.

Manning, S. K. Tactual and visual alphanumeric suffix effects. Quarterly Journal of Experimental Psychology, 1980, 32, 257. 267.

Parkinson, S. R., \& Hubbard, L. L. Stimulus suffix effects in dichotic memory. Journal of Experimental Psychology, 1974, 102, 266-276.

Penney, C. G. Suffix effects in lists of temporally grouped words. Quarterly Journal of Experimental Psychology, 1978, 30, 235250.

Rudel, R. G., \& Denckla, M. B. Relation of forward and backward digit repetition to neurological impairment in children with learning disabilities. Neuropsychologica, 1974, 12, 109118.
Shand, M. A., \& Klima, E. S. Nonauditory suffix effects in congenitally deaf signers of American Sign Language. Journal of Experimental Psychology: Human Learning and Memory, $1981,7,464-474$.

Spoenr, K. T., \& Corin, W. J. The stimulus suffix effect as a memory coding phenomenon. Memory \& Cognition, 1978, 6, 583-589.

\section{NOTE}

1. It should be noted that ceiling effects in backward recall are a possible though unlikely cause of the lack of a large decrement for the most recently presented item. If one assumes a "super image" of this item due to the short time interval between presentation and recall, then the small effect of the suffix on this position could, in fact, represent a drop that is equivalent in true size to the end-of-sequence effect in forward recall. However, the mere existence of a decrement from a stimulus as simple as a suffix suggests that this interpretation is quite unlikely. If a "super image" is present, a simple zero that does not need processing should not interfere at all. Further, the frequent presence in the forward recall paradigm of almost perfect performance on the most recent item, combined with the large end-of-sequence decrement from the suffix, implies the operation of different processes rather than ceiling effects to be the source of the differential size of the suffix effect on the item nearest the suffix in the two recall orders. Additionally, output interference is also an unlikely candidate for the differential suffix effects in forward and backward recall because prefix studies (those in which the redundant not-to-be-recalled item precedes the list) and studies involving running memory span techniques that control for this variable (e.g., Crowder \& Morton, 1969) show end-of-sequence decrements that are different for suffixes than prefixes.

(Received for publication June 14, 1982.) 